\title{
Interaction of membrane-spanning proteins with peripheral and lipid-anchored membrane proteins: perspectives from protein-lipid interactions (Review)
}

\author{
D. Marsh $\dagger^{*}$, L. I. Horváth†, M. J. Swamy§, \\ S. Mantripragada $\uparrow$ and J. H. Kleinschmidt\#
}

†Max-Planck-Institut für biophysikalische Chemie, Abt. Spektroskopie, 37070 Göttingen, Germany

Institute of Biophysics, Biological Research Centre, 6701 Szeged, Hungary

$\S$ School of Chemistry, University of Hyderabad, Hyderabad 5000046, India

-Skye Pharma, San Diego, CA 92121, USA

\#Universität Konstanz, Fachbereich Biologie, 78457

Konstanz, Germany

\section{Summary}

Studies of lipid-protein interactions in double-reconstituted systems involving both integral and peripheral or lipid-anchored proteins are reviewed. Membranes of dimyristoyl phosphatidylglycerol containing either myelin proteolipid protein or cytochrome $c$ oxidase were studied. The partner peripheral proteins bound to these membranes were myelin basic protein or cytochrome $c$, respectively. In addition, the interactions between the myelin proteolipid protein and avidin that was membraneanchored by binding to $\mathrm{N}$-biotinyl phosphatidylethanolamine were studied in dimyristoyl phosphatidylcholine membranes. Steric exclusion plays a significant role when sizes of the peripheral protein and transmembrane domain of the integral protein are comparable. Even so, the effects on avidin-linked lipids are different from those induced by myelin basic protein on freely diffusible lipids, both interacting with the myelin proteolipid protein. Both the former and the cytochrome c/cytochrome oxidase couple evidence a propagation of lipid perturbation out from the intramembrane protein interface that could be a basis for formation of microdomains.

Keywords: Lipid-protein interactions, spin labels, electron spin resonance, myelin proteins, cytochrome oxidase, avidin-biotin.

\section{Introduction}

Electron spin resonance (ESR) with spin-labelled lipids at probe amounts has proved to be one of the most valuable spectroscopic methods for studying lipid-protein interactions in biological membranes (see e.g. Marsh 1985). This is because the dynamic sensitivity of spin-label ESR is optimally matched to the timescale of the rotational motions of the lipid chains in biological membranes, which lies in the ns range. Even if the exchange rate of the lipids at the intramembranous surface of an integral protein is as fast as

*To whom correspondence should be addressed. e-mail: dmarsh@gwdg.de translational diffusion in fluid bilayers, they can be distinguished from the latter in the spin label ESR spectrum. This is possible whenever the lipid rotational mobility differs substantially in the two environments (see figure 1). Then the stoichiometry and specificity of lipid-protein interaction can be determined by using difference spectroscopy to quantitate the 'free' and protein-interacting lipid populations (Marsh 1989).

Analysis of the lipid stoichiometry $\left(N_{b}\right)$ and specificity $\left(K_{r}\right)$ from the two-component ESR spectra uses the equation for equilibrium exchange association. The fraction, $f$, of spinlabelled lipid that is motionally restricted at one of the $N_{b}$ sites at the intramembranous surface of the protein is given by (see Marsh 1985):

$$
f=N_{b} K_{r} l\left[n_{t}+N_{b}\left(K_{r}-1\right)\right]
$$

where $K_{r}$ is the average association constant of the spinlabelled lipid relative to the host lipid and $n_{t}$ is the total lipid/ protein ratio. ESR titrations performed by varying the lipid/ protein ratio confirm that a fixed stoichiometry, $N_{b}$, is maintained independent of $n_{t}$ (Brotherus et al. 1981), and yield values for both $N_{b}$ and the mean relative association constant $K_{r}$. In general, $K_{r} \approx 1$ for a particular spin-labelled lipid (e.g. phosphatidylcholine) relative to its unlabelled parent host lipid. This allows the determination of stoichiometries from measurements at a single lipid/protein ratio.

It is expected that the lipid stoichiometry at the intramembranous perimeter is related directly to the transmembrane structure and degree of oligomerization of the protein (Marsh 1997). Figure 2 compares ESR-determined values of $N_{b}$ with predictions for simple, regular helix packing arrangements. For a single monomeric helix (phospholamban mutant), and for a monomeric 7-helix sandwich (rhodopsin), the predictions work well. For proteolipid hexamers (PLP and 16kD), the stoichiometries per monomer are predictably reduced. For large, highly polytopic proteins such as cytochrome oxidase (or acetylcholine receptor), with more complex and/ or less regular transmembrane arrangements, the simple models do not apply. Further discussion of this topic can be found in Marsh (1997) and Marsh and Horváth (1998).

It is further expected that the selectivity patterns of lipidprotein interaction will reflect in detail the structure and sequence of those sections of transmembrane protein segments that are located in the vicinity of the lipid headgroups. This expectation is borne out in practice by the results of ESR studies. Figure 3 gives the values of $K_{r}$ for representative lipids interacting with a range of different membrane proteins. The lipid selectivity patterns differ for the different proteins. Whereas the highest selectivities are found for anionic lipids, they are not identical for lipids with the same formal charge, nor does a single lipid display the highest selectivity for all proteins. Cytochrome oxidase displays its highest selectivity for cardiolipin, a lipid unique 


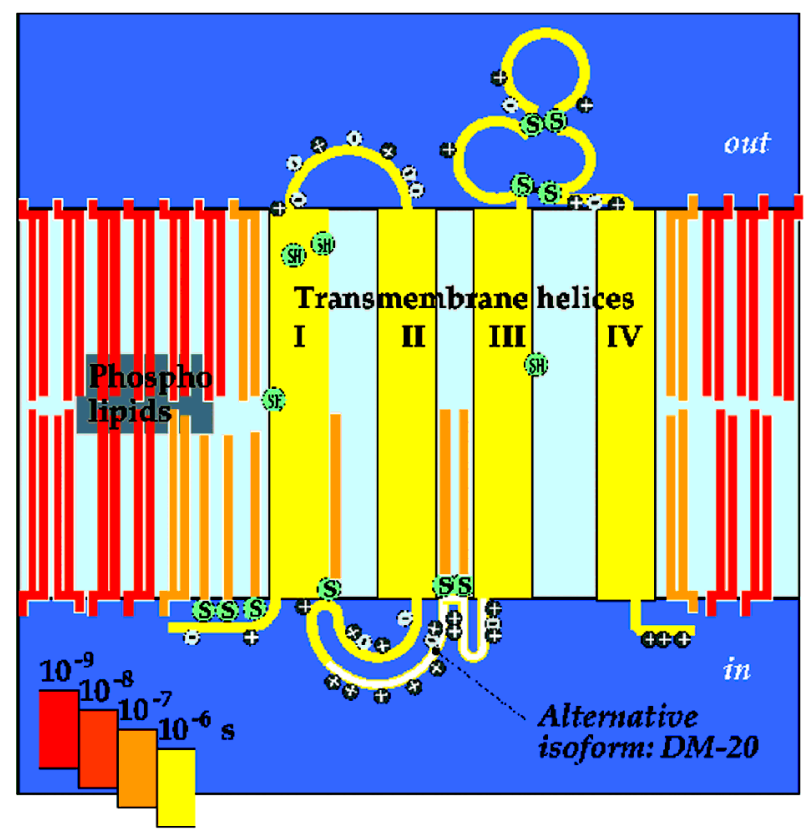

Figure 1. Schematic indication of the rotational mobility of the lipid and protein components in membranes. The grey/colour scale represents the rotational correlation time of the different components. Motional restriction of the spin-labelled lipids in direct contact with the transmembrane four-helix bundle results in a two-component ESR spectrum from which the number of first-shell lipids is quantitated. The transmembrane topology of the myelin proteolipid protein is that determined in Weimbs and Stoffel (1992). The polar loop deleted in the DM-20 isoform is also indicated.

to mitochondria in eucaryotes, whereas rhodopsin displays little selectivity for any particular lipid species. This topic is dealt with further in Marsh (1995) and Marsh and Horváth (1998).

Figures 2 and 3 show that the database on lipid interactions with single integral membrane protein species is reasonably extensive. As already implied, this area has been reviewed several times, one of the most recent being in Marsh and Horváth (1998), which also includes consideration of the penetration of soluble proteins into membranes. A considerable body of work also exists on lipid interactions with single peripheral proteins. Some of this was reviewed in Sankaram and Marsh (1993) and (Marsh (1995). Far fewer biophysical studies have been undertaken on the mutual interactions of integral and peripheral proteins in defined reconstituted systems. This review is devoted specifically to this aspect of protein-lipid interactions, including proteinlinked lipid chains. The latter are considered first, in the absence of a second protein, by using avidin bound to $\mathrm{N}$ biotinyl phosphatidylethanolamine as a model system. Then, the myelin basic protein/myelin proteolipid and cytochrome c/cytochrome oxidase couples are treated, followed finally by lipid-linked avidin interacting with the proteolipid protein.

\section{Avidin/biotin-lipid membrane anchoring}

Phosphatidylethanolamines that are $\mathrm{N}$-derivatised with biotin can specifically bind the protein avidin (see e.g. Swamy and Marsh 2001). Because of the extremely high affinity of avidin

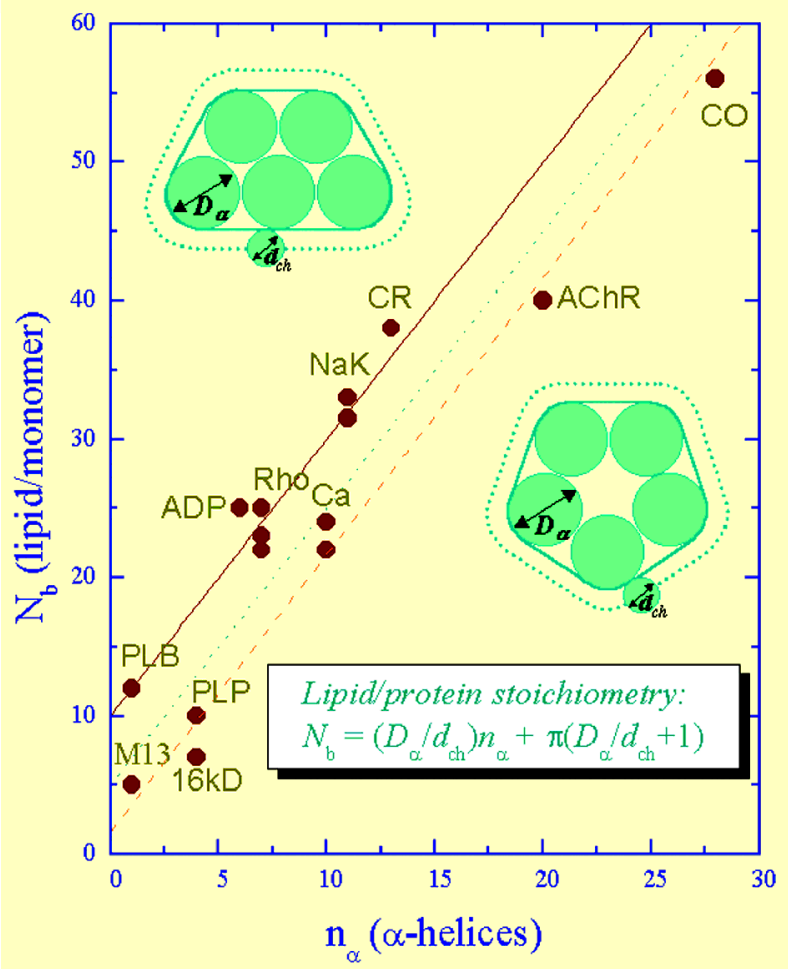

Figure 2. Dependence of the number of first-shell lipids, $N_{b}$ per protein monomer, on the actual or predicted number of transmembrane helices, $n_{\alpha}$, for the following integral proteins: M13, phage coat protein; PLB, L37A mutant of phospholamban; PLP, myelin proteolipid protein; $16 \mathrm{kD}, 16-\mathrm{kDa}$ proteolipid from Nephrops; ADP, ADP-ATP carrier; Rho, rhodopsin; Ca, Ca-ATPase; NaK, Na,KATPase; CR, cytochrome reductase; AChR, acetylcholine receptor; CO, cytochrome oxidase. See Marsh (1997) for references. Predictions for helical sandwiches or regular polygonal arrangements (solid line) are given, where $D_{\alpha}(=1 \mathrm{~nm})$ and $d_{\mathrm{ch}}(=0.48 \mathrm{~nm})$ are the diameters of an $\alpha$-helix and a lipid chain, respectively. Dotted and dashed lines are corresponding predictions for protein dimers and hexamers, respectively.

for biotin, this system serves as a model for covalent lipid anchors. Also, because phospholipases must bind specifically to the lipid headgroups, the avidin/biotin lipid interaction may be used to model certain features of their mode of interaction.

The way in which the lipid chains anchor the water-soluble avidin protein to membranes was studied by using biotinphosphatidylethanolamines (biotin-PE) that are spin-labelled in their sn-2 chain (Swamy and Marsh 1997). These biotinylated lipids were incorporated in membranes of phosphatidylcholine, an inert host lipid that alone does not bind avidin. Figure 4 gives the lipid chain flexibility profile as a function of position, $n$, of spin labelling, and the modification of this profile by binding avidin. A systematic decrease in the spectral outer hyperfine splitting, $A_{\max }$, is found as the spinlabel is stepped down the chain towards the centre of the membrane. This is a measure of the angular amplitude of motional freedom of the lipid chain segments. The characteristic flexibility gradient thus registered by the spinlabelled lipid is preserved on binding avidin to the biotin 


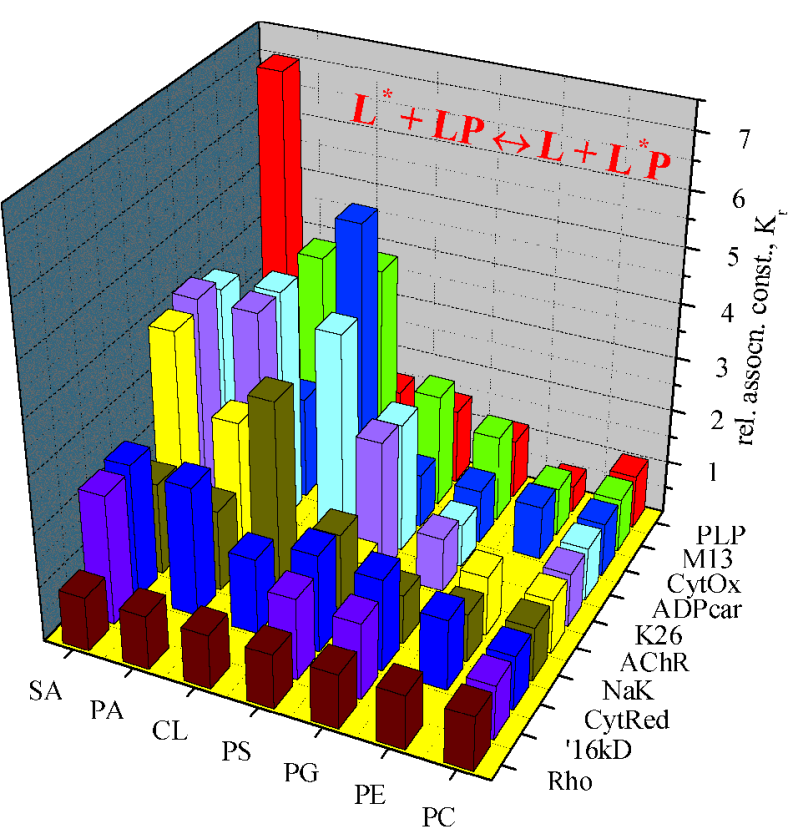

Figure 3. Selectivity patterns for interaction of integral membrane proteins with the following lipids: $P A$, phosphatidic acid; $C L$, cardiolipin; SA, stearic acid; PS, phosphatidylserine, PG, phosphatidylglycerol; PE, phosphatidylethanolamine; PC, phosphatidylcholine. Plotted is the association constant, $K_{r}$, of the various spinlabelled lipid species, relative to the host membrane lipid (usually PC). See Marsh and Horváth (1998) for references.

headgroup, but the overall size of the outer hyperfine splittings increases dramatically. This direct and highly specific effect of the protein-lipid interaction corresponds to an upward shift in the flexibility profile by $\sim 7 \mathrm{CH}_{2}$ units. As indicated in figure 4 , this translates to an upward movement of the biotin lipid, relative to the phosphatidylcholine membrane, by $0.7-0.8 \mathrm{~nm}$. Electron crystallographic studies on two-dimensional arrays of streptavidin bound to biotin lipid have shown that the carboxyl group of the bound biotin is positioned $\sim 0.8 \mathrm{~nm}$ from the surface of the supporting lipid layer (Darst et al. 1991). This is consistent with the vertical movement suggested by the spin-label ESR studies.

Direct evidence for an upward vertical movement of the biotin lipid on complexation with avidin comes from ESR measurements of spin label relaxation enhancement induced by paramagnetic species (Arora and Marsh 1998). The relaxation enhancement of biotin-PE spin labels by aqueous $\mathrm{Ni}^{2+}$ is increased, and that by lipid-soluble $\mathrm{O}_{2}$ is decreased, on binding avidin. The ratio of the $\mathrm{Ni}^{2+} / \mathrm{O}_{2}$ enhancements increases from 0.5 to 2.6 for a spin label on the $\mathrm{C}-8$ atom of biotin-PE, on binding avidin.

This type of vertical displacement is likely to be a general feature of the interaction with proteins, e.g. cholera toxin or anti-cardiolipin antibodies, that bind to lipid headgroups. The enzymes of phospholipid hydrolysis are a further class of examples. In phospholipase $A_{2}$, the active site is located $\sim 1.5 \mathrm{~nm}$ from the protein surface (Scott et al. 1990). A movement of the phospholipid substrate out of the membrane, through a hydrophobic channel that leads to the catalytic site, is, therefore, required for the enzymatic

\section{Spin label position, $n$}

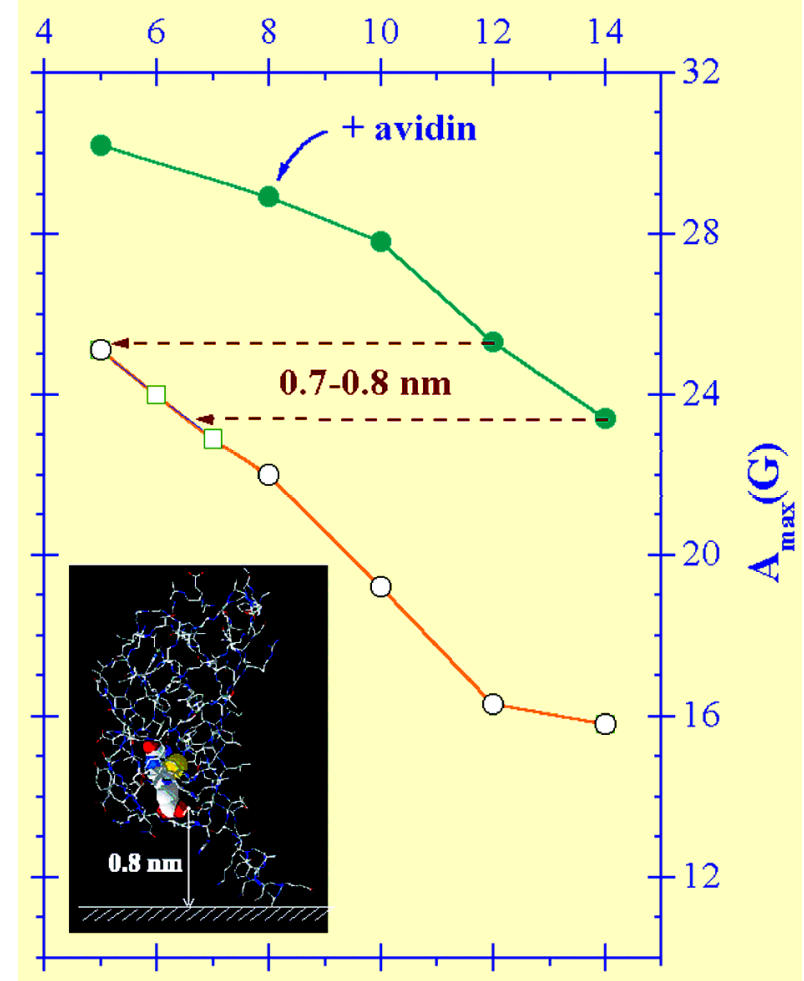

Figure 4. Spin-labelled $N$-biotinyl phosphatidylethanolamine, with (filled circles) and without (open circles) bound avidin, in dimyristoyl phosphatidylcholine membranes. Spin-label outer hyperfine splitting constant, $A_{\max }$, is plotted against spin-label position, $n$, in the $s n-2$ chain. Open squares are for spin-labelled phosphatidylcholine. (Data reproduced with permission from Swamy and Marsh (1997)). The structure of biotin-bound streptavidin is from PDB:1STP (Weber et al. 1989). The location of the lipid surface is approximately that determined in Darst et al. (1991).

hydrolysis. The use of the biotin-PE/avidin couple as a model for a lipid-anchored protein will be returned to in a later section.

\section{Myelin basic protein/myelin proteolipid protein interactions}

Myelin proteolipid (PLP) is the major transmembrane protein and myelin basic protein (MBP) is the major peripheral protein in the membranes of the myelin sheath. Together, they make up $\sim 80 \%$ of the protein of central nervous system myelin, in which they are present in roughly equimolar amounts. The proteolipid protein has a molecular mass of $\sim 25 \mathrm{kDa}$ and is thought to be composed of a four-helix bundle, with the principal polar loop connecting helices II and III (Weimbs and Stoffel 1992). There are three palmitoylation sites in this loop, in addition to three further sites in the $\mathrm{N}$ terminal section. A stretch of 34 contiguous residues (Val116-Lys150) in the II-III loop contains six basic and two acidic residues, together with four histidines; this is deleted in the DM-20 isoform of the proteolipid (see figure 1). The basic protein is a water-soluble protein of molecular 
mass $\sim 18.4 \mathrm{kDa}$, which has little tertiary structure in solution but adopts $\alpha$-helical and $\beta$-sheet structure on binding to membranes (Surewicz et al. 1987). MBP contains 31 positively charged residues that are distributed roughly evenly throughout the sequence, and has a pl of 10.1. In addition, there is a lipid attachment site for phosphatidyl inositol 4,5-biphosphate at residue Ser54, which is also a potential phosphorylation site (Chang et al. 1986).

PLP proteolipid reconstituted in phospholipid bilayer membranes by dialysis from 2-chloroethanol motionally restricts 11 lipids per monomer and displays a pronounced selectivity for various negatively charged phospholipids (Brophy et al. 1984). A large part of this lipid selectivity arises from the polar sequence that is deleted in DM-20 (Horváth et al. 1990). The basic protein (MBP) binds to bilayer membranes that are negatively charged, with a binding stoichiometry of 36 lipids/protein at saturation (Sankaram et al. 1989a). The surface binding of MBP preserves the characteristic flexibility gradient of spinlabelled lipid chains. Mobility is decreased uniformly throughout the length of the chain as a result of the increased lipid packing density induced by surface association of MBP. The resulting increase in ESR spectral anisotropy, $\Delta A_{\max }$, can be used as a measure of binding extent (Sankaram et al. 1989b) and selectivity of lipid interaction (Sankaram et al. 1989c).
Double reconstitutions, involving the binding of MBP to dimyristoyl phosphatidylglycerol (DMPG) membranes that contain PLP, were used to study the mutual protein-lipid interactions of integral and peripheral proteins (Sankaram et al. 1991). Figure 5 gives the saturation binding stoichiometry of MBP (per lipid) as a function of PLP content in the membrane. Up to a critical PLP/DMPG ratio, the binding of MBP is essentially undisturbed by the presence of PLP. Beyond this critical content, the saturation binding of MBP decreases roughly linearly with PLP content, reaching zero at a DMPG/PLP ratio of $N_{1} \approx 11$. The latter corresponds also with the number of lipids $N_{b} \approx 11$ that are motionally restricted by PLP alone (see above). Apparently, this first boundary shell of lipids is unavailable for binding to MBP, once the PLP density in the membrane exceeds a critical value. The critical PLP content corresponds to a DMPG/PLP ratio of $N_{c} \approx 37$. This also corresponds to the number of lipids interacting with MBP at surface saturation (see above). Therefore, the minimum critical area of lipid surface between PLP assemblies that is required for undisturbed binding of MBP is comparable to the size of the basic protein itself. This simple situation of mutual steric exclusion between the two proteins is illustrated schematically in figure 5 . A phenomenological description of the decrease in MBP binding stoichiometry is possible, if proportionality to the total fraction of available

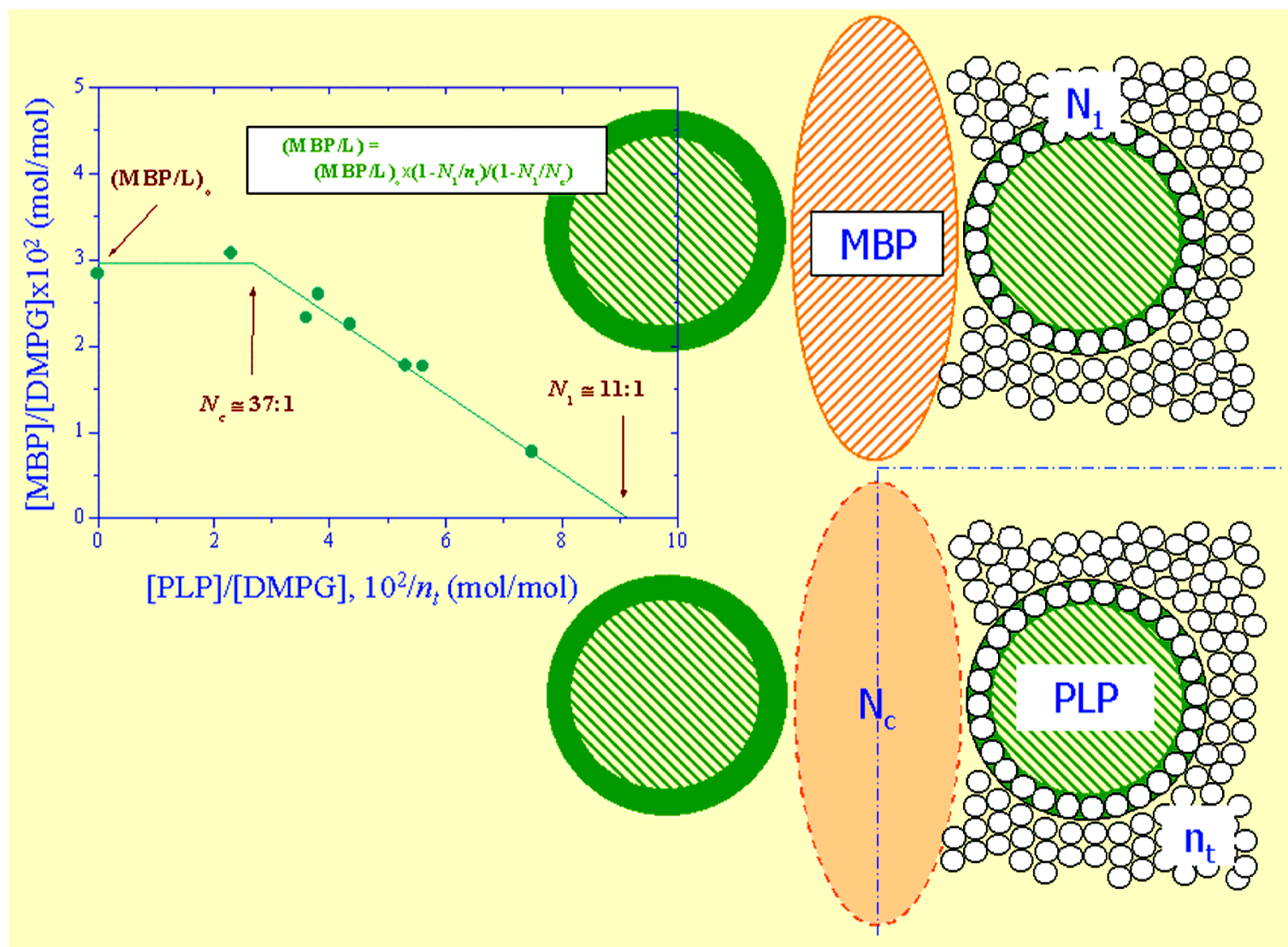

Figure 5. Steric exclusion between the integral proteolipid protein (PLP) and peripheral basic protein (MBP) in dimyristoyl phosphatidylglycerol (DMPG) membranes. A critical number of lipids, $N_{c}$ per PLP, is required for undisturbed surface binding of MBP. For total number of lipids $n_{t}<N_{c}$, MBP binding decreases approximately linearly with $1 / n_{t}$, reaching zero at $n_{t}=N_{1}$. The phenomenological equation given in the inset assumes that 
lipids, viz., $\left(n_{t} N_{1}\right) / n_{t}$, is assumed. This leads to the equation given in the inset to figure 5 and results in the functional dependence shown by the solid lines.

Accompanying the steric exclusion is a reduction by $30-$ $40 \%$ in the stoichiometry of lipids interacting with PLP that is caused by binding of MBP at saturation. This suggests that MBP disturbs the interaction of lipids with the intramembranous portion of PLP, quite possibly by means of the short membrane-penetrant portions of the peripheral protein. Unlike cytochrome $c$, MBP interacts directly with the lipid chains to a limited extent, in addition to the surface electrostatic association (see Sankaram et al. 1989a). In this respect, MBP resembles the precursor protein apocytochrome c (Görrissen et al. 1986).

In addition to steric exclusion between the two proteins, there are also other mutual influences on the lipid-protein interactions. Table 1 gives the increase in spectral anisotropy, $\Delta A_{\max }$, of spin-labelled phosphatidylglycerd on saturation binding of MBP. This effect on lipid mobility is substantially attenuated in the presence of the proteolipid protein. At a DMPG/PLP ratio of $25: 1$, the weakening of the MBP-DMPG interaction is mostly a consequence of the reduced binding of MBP (cf. figure 5). At the lower DMPG/ PLP ratio, the lipid perturbation is reduced less, in spite of further decrease in MBP binding. This undoubtedly results from a mutual reinforcing of the lipid perturbation by both PLP and MBP, at high protein/lipid ratios.

Table 2 gives the effect of MBP binding on the selectivity of lipid interactions with PLP. Selectivities (i.e. $K_{r} / K_{r}{ }^{\mathrm{PC}}$ ) of all lipids for PLP are reduced on binding MBP, particularly the negatively charged ones. This weakening of the selectivity and modification of the selectivity ranking can be understood as a direct competition for the lipids by the two proteins. The relative association constants, $K_{r}$, for interaction of lipids with PLP that are given in table 2 are defined relative to DMPG as standard state in one case, but relative to DMPG with MBP bound in the other.

It, therefore, seems that MBP and PLP probably interact independently, rather than synergistically, in their role in the compaction of nerve myelin. In particular, the double reconstitutions offer little evidence for the direct binding of MBP to PLP that has been suggested in some models.

\section{Cytochrome c/cytochrome oxidase interactions}

Cytochrome $c$ and cytochrome oxidase form the terminal redox couple of the mitochondrial electron transport chain, ending in the reduction of oxygen to water. Eucaryotic cytochrome oxidases are large $\left(M_{r} \sim 200 \mathrm{kDa}\right)$ integral proteins composed of 13 sub-units and containing 28 transmembrane helices. Cytochrome $c$ is a small, watersoluble, basic globular protein of molecular mass $\sim 11.7 \mathrm{kDa}$. Cytochrome oxidase motionally restricts $N_{b}=55$ lipids per $200 \mathrm{kDa}$ protein, at its intramembranous surface (Knowles et al. 1979). It displays a pronounced selectivity for anionic lipids, particularly cardiolipin (Knowles et al. 1981). Cytochrome $c$ binds to negatively charged lipid membranes with a stoichiometry at saturation of nine lipids per bound protein (Görrissen et al. 1986, Sankaram and Marsh 1993). Binding is to the surface of the membrane without appreci-
Table 1. Increase in maximum hyperfine splitting, $\Delta A_{\max }$, of phosphatidylglycerol spin-labelled at the $5-C$ atom of the $s n-2$ chain, on saturation binding of myelin basic protein to membranes of dimyristoyl phosphatidylglycerol (DMPG) containing the myelin proteolipid protein (PLP).

\begin{tabular}{lcc}
\hline & \multicolumn{2}{c}{$\Delta A_{\max }$ (gauss) } \\
\cline { 2 - 3 } DMPG/PLP $(\mathrm{mol} / \mathrm{mol})$ & $10 \mathrm{mM} \mathrm{NaCl}$ & $100 \mathrm{mM} \mathrm{NaCl}$ \\
\hline $1: 0$ & 3.6 & 3.3 \\
$25: 1$ & 1.0 & 0.7 \\
$17: 1$ & 2.3 & 1.2 \\
\hline
\end{tabular}

Values are given for different ionic strengths $(\mathrm{NaCl})$ of the suspending medium and $T=30^{\circ} \mathrm{C}$. Data from Sankaram et al. (1991).

Table 2. Relative association constants, $K_{r}$, of spin-labelled lipids with myelin proteolipid protein (PLP) in dimyristoyl phosphatidylglycerol membranes, with and without myelin basic protein (MBP).

\begin{tabular}{|c|c|c|c|}
\hline \multirow[b]{2}{*}{ Lipid spin label } & \multicolumn{2}{|c|}{$K_{r} / K_{r}^{\mathrm{PC}}$} & \multirow{2}{*}{$\begin{array}{c}\Delta \Delta G_{L} \\
(\mathrm{~kJ} / \mathrm{mol})\end{array}$} \\
\hline & PLP & $\mathrm{PLP}+\mathrm{MBP}$ & \\
\hline stearic acid & 2.9 & 2.3 & 0.6 \\
\hline cardiolipin & 3.0 & 2.7 & 0.3 \\
\hline phosphatidic acid & 2.4 & 1.7 & 0.8 \\
\hline phosphatidylglycerol & 2.0 & 1.8 & 0.3 \\
\hline phosphatidylserine & 1.4 & 1.1 & 0.6 \\
\hline phosphatidylethanolamine & 1.7 & 1.2 & 0.9 \\
\hline
\end{tabular}

$K_{r}$ is normalized to the corresponding value, $K_{r}^{\mathrm{PC}}$, for spin-labelled phosphatidylcholine. $\Delta \Delta G_{L}$ is the increase in free energy of lipid association on binding MBP. Data from Sankaram et al. (1991).

Table 3. Fraction, $f$, of spin-labelled lipid, 14-BPESL (biotin-PE) or 14-PCSL (PC), that is motionally restricted by interacting with PLP (or DM-20) in DMPC membranes (lipid/protein=37:1 $\mathrm{mol} / \mathrm{mol}$ ), in the presence and absence of excess avidin.

\begin{tabular}{llcc}
\hline & & \multicolumn{2}{c}{$f$} \\
\cline { 3 - 4 } Protein & Spin label & - avidin & + avidin \\
\hline PLP & 14-BPESL & 0.51 & 0.80 \\
& 14-PCSL & 0.29 & $-{ }^{a}$ \\
DM-20 & 14-BPESL & 0.57 & 0.79 \\
& 14-PCSL & 0.30 & $-{ }^{a}$ \\
\hline
\end{tabular}

${ }^{a}$ Avidin has no effect on 14-PCSL in DMPC membranes. Data from Swamy et al. (1999).

able penetration of cytochrome $c$ into the hydrophobic core (Kostrzewa et al. 2000).

The mutual interactions of this integral/peripheral protein couple were studied in double reconstitutions, in which cytochrome $c$ is bound to dimyristoyl phosphatidylglycerd membranes containing cytochrome oxidase (Kleinschmidt et al. 1998). The binding of cytochrome $c$ occurs in an unrestricted fashion with a saturation stoichiometry of 9 DMPG per cytochrome $c$ for all realizable cytochrome oxidase contents. This is unlike the situation with the myelin basic protein/proteolipid protein couple already described, because, in this case, the peripheral protein is much smaller than the integral protein (see figure 6). The ratio of the number of lipids per MBP at saturation binding to the number of first-shell lipids motionally restricted by PLP is 36/11 $\sim 3$, whereas the corresponding ratio for cytochrome $c$ and cytochrome oxidase is $9 / 55 \sim 0.2$. With decreasing lipid 
content, steric interactions between cytochrome oxidase molecules become limiting before these can affect the binding of cytochrome $c$ appreciably. Indeed, for efficient electron transfer, cytochrome oxidase must be fully accessible to cytochrome $c$ at the rather high protein packing densities of the mitochondrial inner membrane.
Figure 6 gives the population of motionally restricted lipid, normalized to cytochrome oxidase content, as a function of total lipid (DMPG)/cytochrome oxidase ratio, $n_{t}$. The ordinate is the fraction, $f$, of spin-labelled phosphatidylglycerol that is motionally restricted, multiplied by $n_{t}$. In the absence of cytochrome $c$, this simply gives the number of lipid sites in

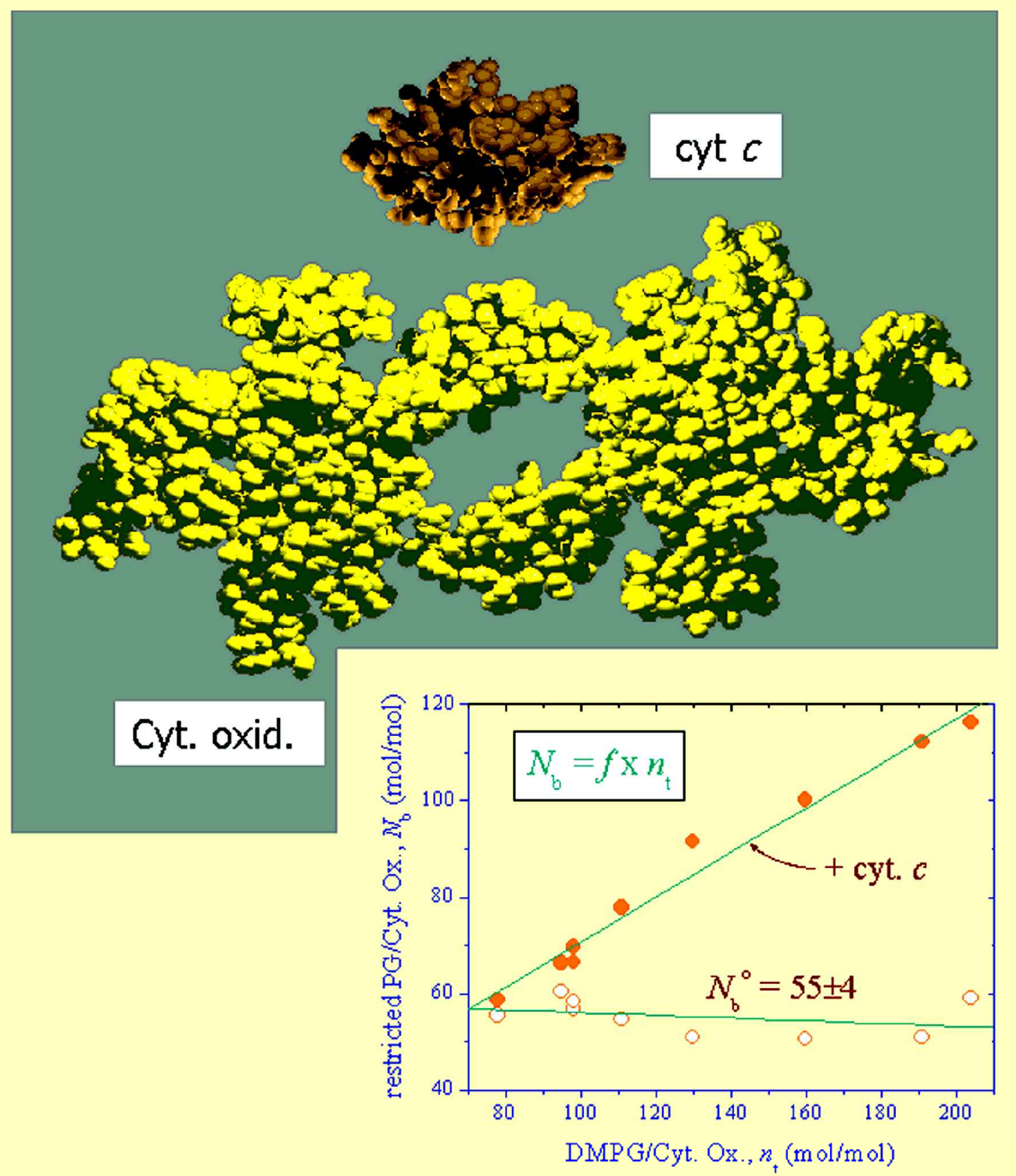

Figure 6. Number of motionally restricted lipids, $N_{b}$ per cytochrome oxidase, in the absence (open circles) and presence (filled circles) of cytochrome $c$ bound at saturation to reconstituted membranes with dimyristoyl phosphatidylglycerol/cytochrome oxidase ratio, $n_{t}$. Assuming $K_{r}=1$ for spin-labelled phosphatidylglycerol, $N_{b}=f \times n_{t}$, where $f$ is the fraction of motionally restricted spin-labelled lipids obtained from the ESR spectrum (data reproduced with permission from Kleinschmidt et al. (1998)). Structure of the transmembrane domain of the cytochrome oxidase dimer is from PDB:1OCC (Tsukihara et al. 1996), and of cytochrome $c$ is from PDB:1AKK (Banci et al. 1997). Orientation of cytochrome $c$ at the membrane surface is that determined in 
the first shell at the intramembranous perimeter of cytochrome oxidase. A constant value of $N_{b}^{\circ}=55$ lipids/cytochrome oxidase, independent of $n_{t}$, is obtained from the data in figure 6. This confirms that $K_{r}=1$ for spin-labelled phosphatidylglycerol relative to DMPG, in accordance with equation (1), and is in quantitative agreement with similar measurements on phosphatidylcholine membranes (Knowles et al. 1979).

In cytochrome oxidase-DMPG membranes saturated with cytochrome $c$, the number of motionally restricted lipids increases progressively with the DMPG/cytochrome oxidase ratio (see figure 6 ). The population of motionally restricted lipid is greater, the greater is the cytochrome c/cytochrome oxidase ratio-at least up to the highest value of $n_{t}$ given in figure 6. Again, this situation is totally unlike that which obtains with the MBP-PLP couple described earlier. In the latter case, the number of lipids that are motionally restricted by PLP is actually reduced by the binding of MBP. The synergistic effect of cytochrome $c$ can be rather large; the motionally restricted lipid population (per cytochrome oxidase) is more than doubled at the highest lipid/protein ratios, $n_{t}$. Therefore, it is most likely that surface-bound cytochrome $c$ propagates the chain restriction induced by cytochrome oxidase to the second and possibly even third shell of lipids surrounding the protein. As already mentioned, cytochrome $c$ binds to the first shell of lipids directly in contact with cytochrome oxidase. Because it binds to about nine DMPG molecules, it could, therefore, form a surface-bridge between lipids in the first and adjacent boundary shells. Such a propagation mechanism is possible because, although cytochrome $c$ alone does not create a specific population of motionally restricted lipids, it does induce a generalized motional restriction of all lipids to which it binds at saturation (Görrissen et al. 1986).

Cytochrome $c$, therefore, can induce formation of microdomains containing cytochrome oxidase in which the mobility of the lipid chains is appreciably restricted relative to that in fluid membranes. Comparison with the MBP-PLP couple suggests that pre-conditions for this mechanism are that the inducing protein binds the lipids directly contacting the integral protein, and possibly also that it does not itself penetrate the membrane. A qualitatively similar phenomenon was observed in the interaction of melittin with the CaATPase and associated lipids in sarcoplasmic reticulum membranes

\section{Avidin-linked chains/proteolipid interactions}

The use of avidin/biotin-PE conjugates as a model for proteins that are anchored to membranes by covalently linked chains, e.g. those of glycosyl phosphatidyl inositol (GPI), was described already. Characterization of the myelin proteolipid as an integral transmembrane protein has also been given. These two different protein classes were used to investigate the interactions of protein-linked lipid chains with transmembrane proteins by using spin-label ESR spectroscopy. Proteolipid (PLP or DM-20) was reconstituted in dimyristoyl phosphatidylcholine (DMPC) membranes in which $\sim 1$ mol\% biotin-PE spin label was incorporated and bound by saturating quantities of avidin (Swamy et al. 1999).
Data given in table 3 show that spin-labelled biotin-PE alone displays a selectivity over phosphatidylcholine for interaction with the proteolipid protein. In part, this may be because biotin-PE is a negatively charged lipid. Saturation binding of avidin to the biotin-PE headgroups has rather dramatic effects that at first sight are perhaps somewhat surprising. It leads to a very substantial increase in the population of motionally restricted biotin-PE chains (see table 3). Approximately $80 \%$ of the avidin-linked chains are restricted in membranes with a DMPC/PLP molar ratio of $n_{t}=37: 1$. This increased population, however, exhibits a lesser degree of chain motional restriction than is typical for first-shell boundary lipids, because it displays a steeper temperature dependence (Swamy et al. 1999).

The relatively high effective stoichiometry of interaction can be explained when allowance is made for the closest interaction distance between the lipid-anchored avidin tetramer and the transmembrane proteolipid hexamers, without any specific interaction between the two types of membrane-associated proteins. As figure 7 indicates, the PLP hexamer and avidin tetramer are of comparable

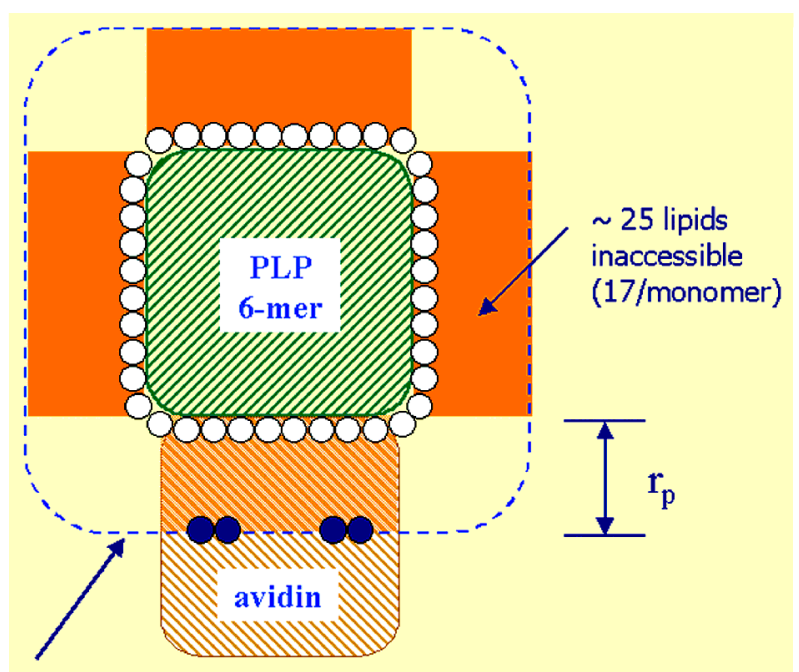

perimeter lipids:

$$
N_{b}=\frac{\pi}{n_{e g g}} \frac{\left(D_{\alpha}+2 r_{p}\right)}{d_{c h}}+\frac{n_{\alpha} D_{\alpha}}{d_{c h}} \approx 15 / \text { monomer }
$$

$$
\begin{aligned}
& \text { fraction of restricted lipids: } \\
& \qquad f \approx \frac{15}{n_{t}-17} \approx 0.75
\end{aligned}
$$

Figure 7. Steric exclusion between the myelin proteolipid hexamer (PLP) and lipid-linked avidin tetramer. The avidin-linked chains ( $)$ can approach only to within distance $r_{p}(\approx 2.5 \mathrm{~nm})$ of the PLP perimeter. The number of lipids, $N_{b}$, that can be accommodated at this distance is given in the figure. $n_{\text {agg }}=6$ is the aggregation number of PLP and other symbols are defined in the legend to figure 2 (adapted with permission from Swamy et al. (1999)). 
cross-sectional area, and the biotin-lipid binding sites are offset from the PLP-lipid interface by $\sim 2.5 \mathrm{~nm}$ (Hendrickson et al. 1989). At this distance, 15 lipids per PLP monomer can be accommodated around the perimeter of the hexamer, compared with $\sim 10$ at the true boundary layer. In addition, the region of lipid bilayer that lies within this extended perimeter is inaccessible to the avidin-linked chains. This region corresponds to approximately half the cross-sectional area of avidin, i.e. $\sim 25$ lipids per avidin tetramer, which translates into $\sim 17$ lipids per PLP monomer (Swamy et al. 1999, Swamy and Marsh 2001). The effective lipid/protein ratio 'seen' by the avidin-linked chains is reduced by this amount and, therefore, the fraction of perimeter chains is given by $f=15 /\left(n_{t}-17\right)=0.75$. This is close to the experimental values of $f$ for 14-BPESL that are given in table 3 . It is, therefore, concluded that, after the geometric extent of the lipid-anchored avidin is taken into account, it displays little selective interaction with the transmembrane proteolipid.

A major consequence of the above interpretation of the increased stoichiometry is that the avidin-linked chains are somewhat removed from the innermost protein-lipid interface. Nevertheless, their mobility is very markedly reduced relative to that in lipid membranes without transmembrane protein, although to a lesser extent than for diffusible firstshell lipids. The effects on the lipid chains of anchoring to avidin (that were discussed in an earlier section) render them far more susceptible to interactions and perturbations within the lipid matrix. This is demonstrated here by the interactions with an integral protein, but may conceivably also extend to interactions with membrane lipids, in particular with the putative raft components sphingolipids and cholesterol.

\section{Conclusions}

The interactions between the two peripheral/integral protein couples considered here differ greatly, being determined by the relative size of the two protein partners and possibly also by their functional roles. Myelin basic protein and proteolipid protein are comparable in size and have a structural role in the compaction of nerve myelin. The interaction between these two membrane proteins is principally one of mutual steric exclusion. Cytochrome $c$ is much smaller than cytochrome oxidase and acts as a substrate of this multisub-unit redox-enzyme complex. The cytochrome associates intimately with its oxidase partner and both reinforces and propagates the influence of the integral protein on the chains of the surrounding lipids. Although the interaction of avidin with the myelin proteolipid protein appears to be one of steric exclusion, the anchoring chains attached to avidin strongly sense the influence of the somewhat remote transmembrane sectors of the proteolipid protein. Thus, one has two rather different mechanisms for propagating lipid interactions induced by transmembrane proteins. Both are protein-driven and involve neither specifically cholesterol nor gel-phase lipid (Schroeder et al. 1994). Nonetheless, both potentially could stabilize membrane microdomains and, hence, be operative in the formation of putative sphingolipid membrane rafts (Simons and Ikonen 1997).

\section{Acknowledgements}

We thank Frau B. Angerstein for the synthesis of spin-labelled phospholipids used in our studies of lipid-protein interactions. We are grateful to $\mathrm{Dr} \mathrm{T}$. Páli for help with figure 6 and Frau I. Dreger for the preparation of the figures. Our studies on lipid interactions with VATPases and the 16-kDa Nephrops protein are supported by the European Union (Contract No. QLGI-CT 2000-01801). DM and JHK are members of the European COST D22 Action.

\section{References}

Arora, A. and Marsh, D., 1998, Protein-induced vertical lipid dislocation in a model membrane system: spin-label relaxation studies on avidin-biotinylphosphatidylethanolamine interactions. Biophysical Journal, 75, 2915-2922.

Banci, L., Bertini, I., Gray, H. B., Luchinat, C., Reddig, T., Rosato, A. and Turano, P., 1997, Solution structure of oxidized horse heart cytochrome c. Biochemistry, 36, 9867-9877.

Brophy, P. J., Horváth, L. I. and Marsh, D., 1984, Stoichiometry and specificity of lipid-protein interaction with myelin proteolipid protein studied by spin-label electron spin resonance. Biochemistry, 23, 860-865.

Brotherus, J. R., Griffith, O. H., Brotherus, M. O., Jost, P. C., Silvius, J. R. and Hokin, L. E., 1981, Lipid-protein multiple binding equilibria in membranes. Biochemistry, 20, 5261-5267.

Chang, P. C., Yang, J. C., Fujitaki, J. M., Chiu, K. C. and Smith, R. A., 1986, Covalent linkage of phospholipid to myelin basicprotein: identification of serine-54 as the site of attachment Biochemistry, 25, 2682-2686.

Darst, S. A., Ahlers, M., Meller, P. H., Kubalek, E. W., Blankenburg, R., Ribi, H. O., Ringsdorf, H. and Kornberg, R. D., 1991, Twodimensional crystals of streptavidin on biotinylated lipid layers and their interactions with biotinylated macromolecules. Biophysical Journal, 59, 387-396.

Görrissen, H., Marsh, D., Rietveld, A. and De Kruijff, B., 1986, Apocytochrome $c$ binding to negatively charged lipid dispersions studied by spin-label electron spin resonance. Biochemistry, 25, 2904-2910.

Hendrickson, W. A., Pahler, A., Smith, J. L., Satow, Y., Merritt, E. A. and Phizackerley, R. P., 1989, Crystal-structure of core streptavidin determined from multiwavelength anomalous diffraction of synchrotron radiation. Proceedings of the National Academy of Sciences (USA), 86, 2190-2194.

Horváth, L. I., Brophy, P. J. and Marsh, D., 1990, Influence of polar residue deletions on lipid-protein interactions with the myelin proteolipid protein. Spin-label ESR studies with DM-20/lipid recombinants. Biochemistry, 29, 2635-2638.

Kleinschmidt, J. H., Powell, G. L. and Marsh, D., 1998, Cytochrome $c$-induced increase of motionally restricted lipid in reconstituted cytochrome $c$ oxidase membranes, revealed by spin-label ESR spectroscopy. Biochemistry, 37, 11579-11585.

Knowles, P. F., Watts, A. and Marsh, D., 1979, Spin label studies of lipid immobilization in dimyristoylphosphatidylcholine-substituted cytochrome oxidase. Biochemistry, 18, 4480-4487.

Knowles, P. F., Watts, A. and Marsh, D., 1981, Spin label studies of headgroup specificity in the interaction of phospholipids with yeast cytochrome oxidase. Biochemistry, 20, 5888-5894.

Kostrzewa, A., Páli, T., Froncisz, W. and Marsh, D., 2000, Membrane location of spin-labelled cytochrome $c$ determined by paramagnetic relaxation agents. Biochemistry, 39, 6066-6074.

Mahaney, J. E., Kleinschmidt, J., Marsh, D. and Thomas, D. D., 1992, Effects of melittin on lipid-protein interactions in sarcoplasmic reticulum membranes. Biophysical Journal, 63, 1513-1522.

Marsh, D., 1985, ESR spin label studies of lipid-protein interactions. In Progress in Protein-Lipid Interactions, Vol. 1, A. Watts and J. J. H. H. M. de Pont, eds. (Amsterdam: Elsevier), pp. 143172.

Marsh, D., 1989, Experimental methods in spin-label spectral analysis. In Biological Magnetic Resonance, Vol. 8, L. J. Berliner and J. Reuben, eds. (New York: Plenum Publishing Corp.), pp. 255-303. 
Marsh, D., 1995, Specificity of lipid-protein interactions. In Biomembranes, A. G. Lee, ed. (Greenwich, CT: JAI Press), pp. 137186.

Marsh, D., 1997, Stoichiometry of lipid-protein interaction and integral membrane protein structure. European Biophysics Journal, 26, $203-208$.

Marsh, D. and Horváth, L. I., 1998, Structure, dynamics and composition of the lipid-protein interface. Perspectives from spin-labelling. Biochimica et Biophysica Acta, 1376, 267-296.

Sankaram, M. B. and Marsh, D., 1993, Protein-lipid interactions with peripheral membrane proteins. In New Comprehensive Biochemistry, Vol. 25. Protein-Lipid Interactions, A. Watts, ed. (Amsterdam: Elsevier), pp. 127-162.

Sankaram, M. B., Brophy, P. J. and Marsh, D., 1989b, Interaction of two complementary fragments of the bovine spinal cord myelin basic protein with phospholipid bilayers. An ESR spin label study. Biochemistry, 28, 9692-9698.

Sankaram, M. B., Brophy, P. J. and Marsh, D., 1989c, Selectivity of interaction of phospholipids with bovine spinal cord myelin basic protein studied by spin-label electron spin resonance. Biochemistry, 28, 9699-9707.

Sankaram, M. B., Brophy, P. J. and Marsh, D., 1989a, Spin label ESR studies on the interaction of bovine spinal cord myelin basic protein with dimyristoylphosphatidylglycerol dispersions. Biochemistry, 28, 9685-9691.

Sankaram, M. B., Brophy, P. J. and Marsh, D., 1991, Lipid-protein and protein-protein interactions in double recombinants of myelin proteolipid apoprotein and myelin basic protein with dimyristoylphosphatidylglycerol Biochemistry, 30, 5866-5873.

Schroeder, R., London, E. and Brown, D., 1994, Interactions between saturated acyl chains confer detergent resistance on lipids and glycosylphosphatidylinositol(GPI)-anchored proteins: GPI-anchored proteins in liposomes and cells show similar behavior. Proceedings of the National Academy of Sciences (USA), 91, 12130-12134.
Scott, D. L., White, S. P., Otwinowski, Z., Yuan, W., Gelb, M. H. and Sigler, P. B., 1990, Interfacial catalysis: the mechanism of phospholipase $A_{2}$. Science, 250, 1541-1546.

Simons, K. and Ikonen, E., 1997, Functional rafts in cell membranes. Nature, 387, 569-572.

Surewicz, W. A., Moscarello, M. A. and Mantsch, H. H., 1987, Fourier transform infrared spectroscopic investigation of the interaction between myelin basic protein and dimyristoyl phosphatidylglycerol bilayers. Biochemistry, 26, 3881-3886.

Swamy, M. J. and Marsh, D., 1997, Spin-label studies on the anchoring and lipid-protein interactions of avidin with $N$ biotinylphosphatidylethanolamines in lipid bilayer membranes. Biochemistry, 36, 7403-7407.

Swamy, M. J. and Marsh, D., 2001, Specific surface association of avidin with $N$-biotinylphosphatidylethanolamine membrane assemblies: effect on lipid behavior and acyl-chain dynamics. Biochemistry, 40, 14869-14877.

Swamy, M. J., Horváth, L. I., Brophy, P. J. and Marsh, D., 1999, Interactions between lipid-anchored and transmembrane proteins. Spin-label ESR studies on avidin-biotinyl phosphatidylethanolamine in membrane recombinants with myelin proteolipid protein. Biochemistry, 38, 16333-16339.

Tsukihara, T., Aoyama, H., Yamashita, E., Tomizaki, T., Yamaguchi, H., Shinzawa-Itoh, K., Nakashima, R., Yaono, R. and Yoshikawa, S., 1996, The whole structure of the 13-subunit oxidized cytochrome $c$ oxidase at $2.8 \AA$. Science, 272, 1136-1144.

Weber, P. C., Ohlendorf, D. H., Wendoloski, J. J. and Salemme, F. R., 1989, Structural origins of high-affinity biotin binding to streptavidin. Science, 243, 85-88.

Weimbs, T. and Stoffel, W., 1992, Proteolipid protein (PLP) of CNS myelin: positions of free, disulfide-bonded, and fatty acid thioester-linked cysteine residues and implications for the membrane topology of PLP. Biochemistry, 31, 12289-12296. 